\title{
Distribution, density and abundance of Antarctic ice seals off Queen Maud Land and the eastern Weddell Sea
}

\author{
Eliezer Gurarie $^{1,2}{ }_{(1)} \cdot$ John L. Bengtson ${ }^{1} \cdot$ Marthán N. Bester $^{3,4} \cdot$ Arnoldus Schytte Blix $^{5}$ • \\ Michael Cameron ${ }^{1} \cdot$ Horst Bornemann ${ }^{6}$ Erling S. Nordøy ${ }^{5} \cdot$ Joachim Plötz $^{6}$. \\ Daniel Steinhage $^{6} \cdot$ Peter Boveng $^{1}$
}

Received: 18 December 2015/Revised: 26 July 2016/Accepted: 15 August 2016/Published online: 9 September 2016 (c) The Author(s) 2016. This article is published with open access at Springerlink.com

\begin{abstract}
The Antarctic Pack Ice Seal (APIS) Program was initiated in 1994 to estimate the abundance of four species of Antarctic phocids: the crabeater seal Lobodon carcinophaga, Weddell seal Leptonychotes weddellii, Ross seal Ommatophoca rossii and leopard seal Hydrurga leptonyx and to identify ecological relationships and habitat use patterns. The Atlantic sector of the Southern Ocean (the eastern sector of the Weddell Sea) was surveyed by research teams from Germany, Norway and South Africa using a range of aerial methods over five austral summers between 1996-1997 and 2000-2001. We used these observations to model densities of seals in the area, taking into account haul-out probabilities, survey-specific sighting probabilities and covariates derived from satellite-based ice
\end{abstract}

Electronic supplementary material The online version of this article (doi:10.1007/s00300-016-2029-4) contains supplementary material, which is available to authorized users.

Eliezer Gurarie

egurarie@umd.edu

1 Marine Mammal Laboratory, NOAA Alaska Fisheries Science Center, 7600 Sand Point Way N.E, Seattle, WA 98115 , USA

2 Present Address: Department of Biology, University of Maryland, College Park, MD 20742, USA

3 Department of Zoology and Entomology, Mammal Research Institute, University of Pretoria,

Private Bag X20, Hatfield, Pretoria 0028, South Africa

4 Hanse-Wissenschaftskolleg, Lehmkuhlenbusch 4, 27753 Delmenhorst, Germany

5 Department of Arctic and Marine Biology, UiT The Arctic University of Norway, Troms $\varnothing$, Norway

6 Alfred-Wegener-Institut, Helmholtz-Zentrum für Polar- und Meeresforschung, Am Handelschafen 12, 27570 Bremerhaven, Germany concentrations and bathymetry. These models predicted the total abundance over the area bounded by the surveys $\left(30^{\circ} \mathrm{W}\right.$ and $\left.10^{\circ} \mathrm{E}\right)$. In this sector of the coast, we estimated seal abundances of: $514\left(95 \%\right.$ CI 337-886) $\times 10^{3}$ crabeater seals, $60.0(43.2-94.4) \times 10^{3}$ Weddell seals and $13.2(5.50-39.7) \times 10^{3}$ leopard seals. The crabeater seal densities, approximately 14,000 seals per degree longitude, are similar to estimates obtained by surveys in the Pacific and Indian sectors by other APIS researchers. Very few Ross seals were observed ( 24 total), leading to a conservative estimate of 830 (119-2894) individuals over the study area. These results provide an important baseline against which to compare future changes in seal distribution and abundance.

Keywords Crabeater seal · Leopard seal · Ross seal · Weddell seal · Antarctic Pack Ice Seal Program (APIS)

\section{Introduction}

Ice-associated seals in Antarctica are considered to be highly abundant (Reeves and Stewart 2003), yet absolute numbers as well as ecological relationships between species and habitat remain poorly known primarily due to the logistical difficulty of studying them in their remote and inaccessible areas. Four species of seal compose the Antarctic 'ice seals', all members of the Lobodontini: the crabeater seal (Lobodon carcinophaga), Weddell seal (Leptonychotes weddellii), Ross seal (Ommatophoca rossii) and leopard seal (Hydrurga leptonyx).

Three of these species are most strongly associated with the pack ice: the crabeater seal (by far, the most abundant), the Ross seal and the leopard seal, while the Weddell seal primarily inhabits the shore-fast ice. Numerous surveys of 
these seal species, primarily from shipboard surveys, were conducted between 1968 and 1983 (Erickson and Hanson 1990; Bengtson et al. 2011). Based on these surveys, a minimum circumpolar abundance of 7 million crabeater seals was reported, along with point estimates of approximately 800,000 Weddell seals, 300,000 leopard seals and 132,000 Ross seals (Erickson and Hanson 1990). These estimates were based on reviewing and aggregating many surveys, most of which did not report precision estimates and were susceptible to biases related to nonoptimal placement of transects (i.e. shipboard surveys conducted along the ice edge, rather than across gradients of ice coverage). The estimates also did not account for the extent of ice, simply because those data were unavailable at a continental scale until the advent of satellite-based remote sensing, and used coarse corrections for haul-out behavior, which have similarly improved with telemetry studies. Finally, these estimates relied on a multi-decade span of survey effort, during which conditions and abundances may well have changed.

The Antarctic Pack Ice Seals (APIS) Program was a coordinated international program of ship and aerial seal surveys designed to deal with the drawbacks of previous efforts by unifying a synoptic, circumpolar survey effort of the four Antarctic pack ice seal species (Southwell et al. 2012). In particular, the survey effort was meant to be as simultaneous and methodologically uniform as possible, with estimates improved by accounting for haul-out probabilities, distance-dependent sampling, and accounting for the dependence of densities on environmental and oceanographic covariates like ice coverage and bathymetry. Pack ice seal abundance derived from the APIS effort have been reported for large portions of Antarctica, including Australian efforts in East Antarctica (ca $40-120^{\circ}$ E Southwell et al. 2008a, b, c), US efforts in the Amundsen and Ross Seas (ca $150^{\circ} \mathrm{E}-100^{\circ} \mathrm{W}$ Bengtson et al. 2011) and UK efforts in the West Antarctic Peninsula and Western Weddell Sea (ca $85-45^{\circ} \mathrm{W}$ Forcada et al. 2012).

Here, we report on survey efforts conducted by separate German, Norwegian and South African surveys between 1996 and 2001. These surveys covered roughly one-third of the Antarctic coastline corresponding to the eastern Weddell Sea and the coast of Queen Maud Land (ca $27.5^{\circ} \mathrm{W}-$ $7.5^{\circ} \mathrm{E}$, Fig. 1), and the results presented here largely complete the intended circumpolar survey coverage of the APIS Program.

There were some significant differences in the methodologies of these three research groups: The German survey was based on closely spaced video-based airplane transects with no identification to the species level, while the Norwegian and South African teams performed visual surveys using helicopters flown from ships along the ice edge. Nonetheless, we unified these observations to model ice seal densities against remotely sensed ice and bathymetric covariates, and used these models to make estimates of total abundance of ice seals along an approximately $1700 \mathrm{~km}\left(40^{\circ}\right.$ longitude) stretch of the Antarctic coast, from $30^{\circ} \mathrm{W}$ to $10^{\circ} \mathrm{E}$.

\section{Materials: survey methods and preliminary processing}

\section{Norwegian survey}

Norwegian research focused on distribution, dive behavior and population abundance of crabeater, Ross, Weddell and leopard seals in the Weddell Sea and in the pack ice off the coast of Queen Maud Land (Nordøy et al. 1995; Blix and Nordøy 2007; Nordøy and Blix 2009). Three expeditions were conducted: NARE (Norwegian Antarctic Research Expeditions) 1992/93, NARE 1996/97 and NARE 2000/01. Here we analyze the data from the 1996/97 survey, which was conducted between January 20 and February 20, 1997 (Fig. 1).

The Norwegian survey was conducted from a ship-based helicopter (Aerospatiale AS Ecureuil 350 B), flown at $91 \mathrm{~m}$ $(300 \mathrm{ft})$. In most transects there were three observers, one at each rear side window (right and left) of the helicopter, and one at the front. Sightings of seals were attributed to specific distance bins identified by marking the back seat windows on each side of the helicopter with red horizontal stripes. Accurate distances between stripes were measured on both sides with Bin 1 starting at the bottom and Bin 7 at the top. Bin 0 was the area below the helicopter, not visible for the Left/Right observer. The head of each observer was fixed during observations by use of a wooden stick attached to the edge of a flat head cap. The end of the stick was placed at the window on a specific spot in such a way that the observer's head could "rest" against the window in a relatively fixed position, thus making the area observed through the space between the marked window stripes constant. Distance between window and forehead was 21.4 and $21 \mathrm{~cm}$ for the left and right observer, respectively.

The area covered by each distance bin at each side was calibrated on an airport in Norway after return from the expedition. The helicopter hovered at a fixed position at the end of the $1000 \mathrm{~m}$ runway at $91 \mathrm{~m}(300 \mathrm{ft})$ altitude, while a person on the ground placed markers on the runway for every transition between distance bins (on instructions from the observer by use of an intercom), as observed by the right/left observer looking through the back seat windows marked with distance stripes. Distance on the ground between markers was then accurately measured by use of a Trumeter (Geo Fennel, Germany) distance wheel. 


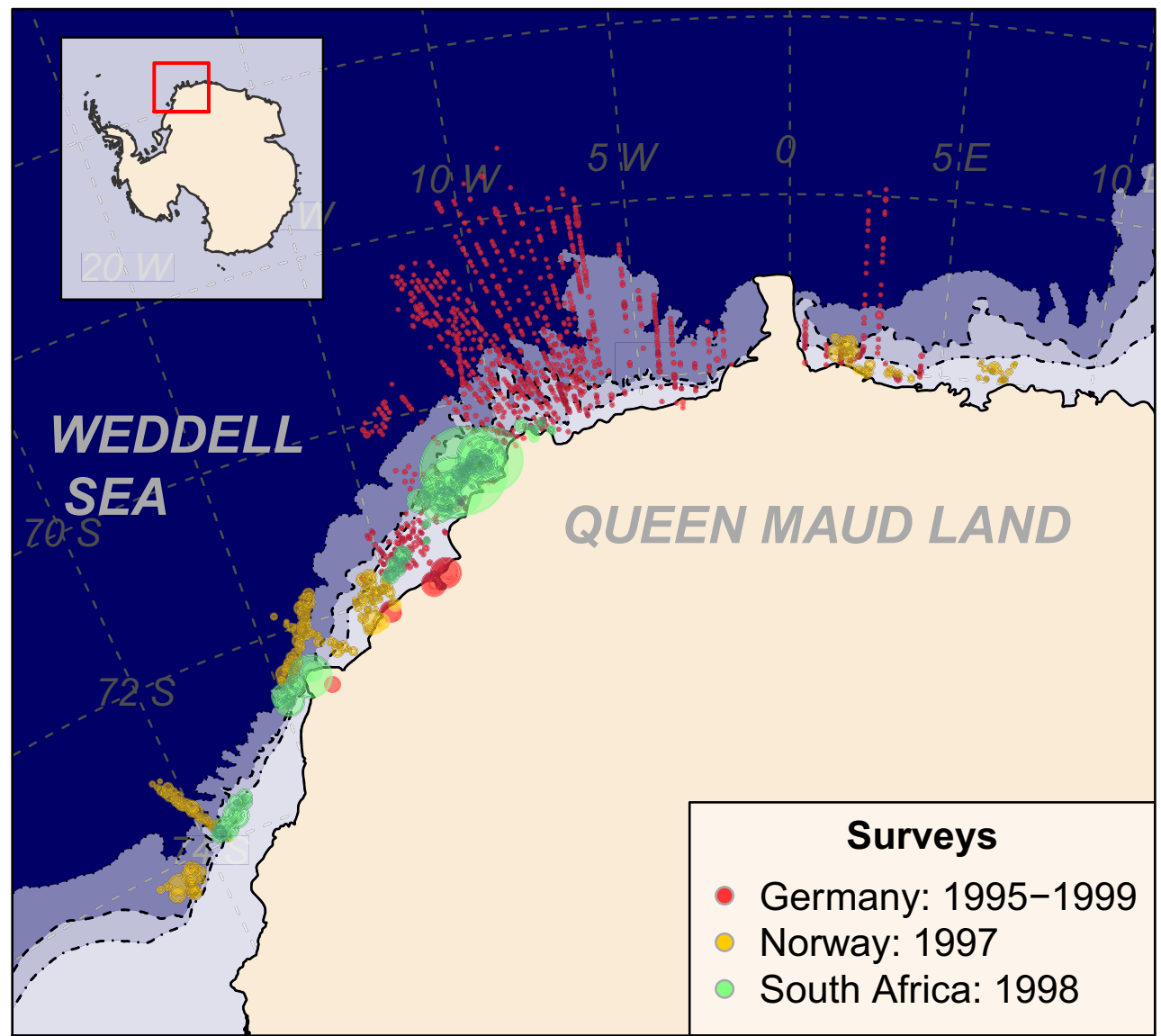

Fig. 1 Map of study area with all seal observations for the three country surveys, color coded as per the legend. Area of circles are proportional to the number of seals per sighting, ranging from 1

A total of 13 transects were flown, of which the first two were test surveys during which the observers experimented with measuring angles of sighting and distance bins. Thus the data used in this analysis were constrained to Transects 3-11 (Fig. 1, online resource A.1). Flights were conducted in the late morning and early afternoon (median 11:00, inter quartile range 10:00-12:00). The maximum observable distance of Bin 7 was set to 1.8 and $2.2 \mathrm{~km}$ for the left and right sides of the plane (Table A3, Fig. 2).

\section{South African survey}

The South African survey was conducted in cooperation with the German APIS/EMAGE project (see following section) during the 1997/1998 summer cruise of the RV Polarstern between January 24 and March 7, 1998 in an area along the northeastern end of the Weddell Sea bounded between $24.4^{\circ}$ and $8.1^{\circ} \mathrm{W}$ and $73.9^{\circ}$ and $70.4^{\circ} \mathrm{S}$ latitude (Fig. 1). Fifteen survey helicopter flights were flown, alternating between a Bell Long Ranger II and Bölkow Blohm 105 with, mostly, two observers, one on either side of the plane. Perpendicular distance from the transect line (smallest dots) to 899 (largest dot in South African survey). Increasingly pale blue colors represent the 3000, 2000 and $1000 \mathrm{~m}$ depth contours

to the center of each seal group was measured in one of six distance bins at 10 degree intervals between 30 degrees from the vertical to the horizon. For two different altitudes (61 and $91 \mathrm{~m}$ ), different midpoints were calculated for each bin using the curved distance along the surface of the earth or intercepting rays at the appropriate angles. Because bin 6 technically reaches the horizon, but sightings at such great distances are not feasible, the maximum distance of the sixth bin was truncated to 667 and $1000 \mathrm{~m}$ for 61 and $91 \mathrm{~m}$ altitudes, respectively (Table A4, Fig. 2). For further details see Bester and Odendaal (1999, 2000).

The first flight was a test survey and therefore excluded from the analysis. The two final flights (numbers 14 and 15) were conducted well to the northwest of this region, near the tip of the Antarctic Peninsula and therefore also excluded from this analysis (Online Resource A.1). Thus, we used data collected from 12 flights (numbered 2-13) conducted between January 24 and February 22, 1998 off Queen Maud Land. Because the winter of 1997/98 was an exceptionally low ice year, the surveyed area had considerably less ice than in the year of the Norwegian surveys, leading to generally shorter flights. 
Fig. 2 Detection probabilities for a the Norwegian survey and b the South African survey with detection bins (shaded gray bars) and midpoints (dots). In the Norwegian survey (a) the detection on the left and right side of the plane are represented and the effective strip width is the vertical dotted lines. In the South African survey (b), the detection probability at 92 and $61 \mathrm{~m}$ respectively is presented, with the vertical dotted line indicating the estimated halfstrip width at each altitude. All species were pooled for the detection estimations, and the assumption was made that the peak bin corresponded to $100 \%$ detection
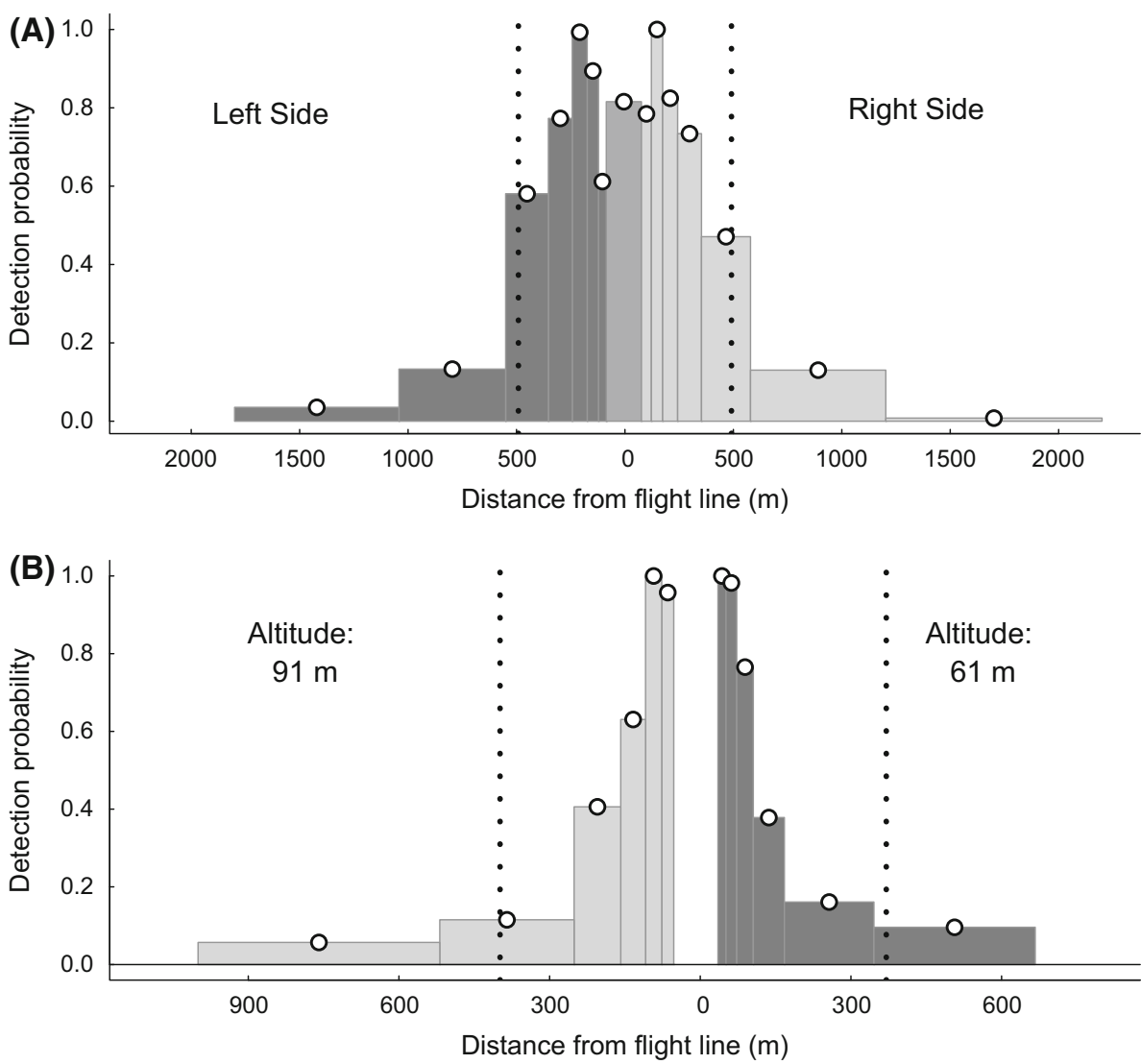

The 12 surveys used in the analysis were all flown between 10:00 and 14:00, with distances ranging from 23.4 to $160.1 \mathrm{~km}$ (mean 90.5 , sd $=39.9$ ), for a total distance of $1085.7 \mathrm{~km}$. Most were flown at an altitude of $61 \mathrm{~m}$ and a speed of 60 knots (total distance: $819 \mathrm{~km}$ ), except the first two (flights 2 and 3) which were flown at $91 \mathrm{~m}$ and at 80 knots (total distance: $267 \mathrm{~km}$ ). Each flight was subdivided into a number of blocks, defined by the presence of seals, i.e., there were no empty blocks, and blocks were of variable length (mean $15.97 \mathrm{~km}$, range 1.55-44.42 km). There were a total of 68 blocks between the 12 flights (ranging from 1 block in flight 6 to 11 blocks in flight 9). Within each block, seals were counted from windows on both sides of the helicopter, except for four flights (numbers 2, 3, 9 and 10) where observations were only taken from one side. Wherever possible, seal species were identified and group sizes were recorded. The South African seal census data are available via the data publisher PANGAEA (Bester et al. 2016).

\section{German surveys}

German surveys were performed over five consecutive austral summers from 1996/97 to 2000/01 in the eastern part of the Weddell Sea (Fig. 1, Online Resource A.1). Seal observations were collected in the context of unrelated aeromagnetic experiments (East Antarctic Margin Aeromagnetic and Gravity Experiment, EMAGE), which provided the platform for digital video recordings of the sea ice and water surface. The digital video camera was mounted on a fixed-wing Dornier DO228-101 aircraft ("Polar 2"). The camera (Sony DCR-VX 1000 E, 1:1.6 zoom lens, f:5.9-59 mm) was fixed on a mount inside the aircraft and pointed vertically down through a doubleglazed hole in the bottom of the plane. The altitude of the aircraft was $152 \mathrm{~m}$ and the speed $240 \mathrm{~km} / \mathrm{h}$. The majority of survey transects were flown in a NW-SE direction approximately perpendicular to the coastline. Spacing between the flight transects was approximately $10 \mathrm{~km}$ (Fig. 1).

Most surveys were conducted between 10:00 and 18:00 UTC (mean 13:45). Strip widths varied on the flights and were recorded (most commonly $70 \mathrm{~m}$; otherwise 30, 50, 80 or $120 \mathrm{~m}$ ). The strip widths were derived from the results of calibrations on land where the camera was pointed at reference objects at predefined altitudes near Neumayer Station II. The quality of video images was sufficient for identifying the presence and group sizes of seals hauled out on pack ice. However, the resolution on the footage was not sufficient for identifying seal species from the video images. Only 37 out of 1334 sightings were assigned species identification, all on a single survey, and this 
sample size was too low for modeling relative abundances. Thus all analyses were conducted based on sightings of seals pooled across species. While groups of seals were likely groups of the same species, some sightings may have been composed of several different species on a single transect. Transect coordinates (lat/lon) were determined for every seal detected on the video based on the time stamp from the camera linked to the airplane's GPS and flight log (UTC). Physical features such as sea ice coverage, ice shelf/edge, fast ice, coastal polynya, pack ice and the northern sea ice margin were also determined from the video footage. The German seal census primary data are available via the data publisher PANGAEA (Plötz et al. 2011a, b, c, d, e).

\section{Correcting for distance-dependent detection}

In the German surveys, detectability of seals was assumed to be $100 \%$ across the field of the video capture, which was more narrow than typical visual observations in distance-based transect methods but uniform in detection probability.

The Norwegian and South African surveys, in contrast, were based on visual observation. Probability of detection was lower at large distances due to smaller apparent sizes of the targets and the greater likelihood of a target being obscured by broken ice and hummocks. Sometimes, the bins closest to the transect line also had lower than peak detection due to the high speed and more awkward angle of observations.

We defined a single effective strip width $W^{*}$ for a survey as the strip for which the same number of seals would have been observed assuming $100 \%$ detection. Thus:

$N=L \sum_{i=1}^{k} d_{i} w_{i}=L W^{*} D^{*}$

where $N$ is the total number of seals observed, $k$ is the number of bins, $L$ is the total length of the survey, $w_{\mathrm{i}}$ is the width of the $i$ th strip, $d_{\mathrm{i}}$ is the density within the $i$ 'th strip, and $D^{*}$ is the aggregated true density of seals. This expression can be rewritten as

$W^{*}=\sum p_{i} w_{i}$

where $p_{i}=d_{i} / D^{*}$ is the probability of detection within the $i$ th strip. We used this relationship to estimate effective strip widths for both the Norwegian and South African surveys, in both cases pooling all of the seal species including the unidentified seals to maximize sample sizes.

In the Norwegian survey, where different bin widths were reported for the left and right sides of the helicopter, we calculated the density of seals observed in each of the 15 bins (seven on the left, seven on the right and one in the middle), pooling the four species and including unidentified seals (which were otherwise excluded from the analysis). We assumed that the bin with the highest density represented $100 \%$ detection, and thus $D^{*}$. We normalized the remaining densities according to $D^{*}$ and solved Eq. 2 . The highest density was observed on the second bin to the right $\left(.217\right.$ seals $\left./ \mathrm{km}^{2}\right)$, very close to the third bin on the left $\left(0.215\right.$ seals $/ \mathrm{km}^{2}$, Fig. 2a). The final estimated effective strip width was $982.5 \mathrm{~m}$.

In the South African survey the same bin widths were reported for both sides of the aircraft. We therefore pooled the counts from each side and estimated a half-strip width using the relationship in Eq. 2. However, because the surveys were conducted at two different altitudes effective half-strip widths were estimated for each altitude (Fig. 2b). The final strip widths were $399 \mathrm{~m}$ at 91 and 370.4 at $61 \mathrm{~m}$. The smaller strip width of the South African survey compared to the Norwegian survey is explained, in part, by the fact that the ice in 1998 was more compacted and dense. At decreasing angles more and more seals were hidden behind packed ice or hummocks, dramatically decreasing the detection probability at greater distances.

\section{Correcting for haul-out probability}

We corrected the densities according to modeled probabilities of hauling out as a function of time of day and day of year $P_{h}$ (Hour, Day). For each species of seal, we used the best available species-specific data on haul-out behavior.

For crabeater seals, the correction was based on analysis of time spent in and out of the water by satellite-tagged seals from Bengtson et al. (2011). The lowest probabilities of being hauled out occur between 22:00 and 24:00. The majority ( $82 \%$ ) of flights took place between 11:00 and 17:00 (locally adjusted time), such that most of the sighting haul-out probabilities were between 0.5 and 0.8 . However the few flights outside of this range led to some estimated haul-out probabilities as low as $2 \%$. We used the same haul-out model for all the German data and for the crabeater seals observed in the Norwegian and South African surveys.

For Weddell seals, we used time-depth records from 27 individuals monitored in Drescher Inlet $\left(72^{\circ} 50^{\prime} \mathrm{S}\right.$, $19^{\circ} 26^{\prime} \mathrm{W}$ ) in 1995 (4 females, 4 males), 1998 (10 females, 5 males), and 2003 (4 females). Hourly haul-out percentages were estimated from multiple readings of the pressure transducers (7.5-60 readings $\mathrm{min}^{-1}$, depending on the instrument version), counting all 'surface' readings as hauled out. This may have biased haul-out time slightly upward because seals sometimes remain in the water, in breathing holes, recovering from long dives. The hourly haul-out percentages were related to hour of the day (UTC) 
and day of the year using a regression method that accounts for autocorrelation (Ver Hoef et al. 2010). Only time of day was a significant covariate of haul-out percentage (figure A7).

Diving behavior of leopard seals in the region of the survey was studied by Nordøy and Blix (2009), who estimated haul-out probabilities in February peaking midday at around 0.40 , a blanket correction that we applied to all leopard seal estimates. Blix and Nordøy (2007) similarly examined the diving behavior of eight Ross seals off Queen Maud Land, determining that the haul-out probability over the time periods of both surveys (early February) was a relatively high 0.65 , which we then used as a correction factor for Ross seal estimates.

Depending on the species, we either applied the correction factors to the raw observations or to the fitted densities as detailed in the modeling section below.

\section{Environmental predictors of seal density}

We used available remotely sensed ice data and oceanographic models to model the observed densities of seals and then to apply those models over the entire range of the surveys.

Ice concentrations were obtained from Special Sensor Microwave/Imager (SSM/I) satellite-derived data, converted and gridded by the National Sea Ice Data Center (NSIDC, Boulder, CO) (Comiso 1990). These data are freely downloadable from the NSIDC website (http://nsidc. org/data/docs/daac/nsidc0002_ssmi_seaice.gd.html) and provide a $25 \times 25 \mathrm{~km}$ grid of ice concentration estimates obtained from brightness temperatures dating to 1979 . Because the daily data did not always provide complete coverage, we used a three-day moving average ice concentration for each grid point. The three-day average provided nearly complete coverage of the grid points in the survey area and reduced noise in the sea ice concentration data.

From the ice concentration grid, we computed four variables for further modeling: the SSM/I sea ice concentration (Ice, ranging from 0 to 100 in \%), the distance of each seal observation to ice edge (DEdge), defined as the $10 \%$ ice contour line, an ice extent metric (IceExtent) calculated as the perpendicular distance of the nearest ice edge to the shore for each sighting, and an ice flux metric (dIceExtent), defined as the difference in IceExtent at a given moment from its value one week prior. This variable quantified the rate of contraction (or, more rarely, expansion) across the regions, and was introduced to test the mechanistic hypothesis that in those regions where the ice extent was contracting more rapidly, there would be a higher concentration of seals closest to the ice edge.
The bathymetry covariate was obtained from the ETOPO1 Global Relief Model provided by the NOAA National Geophysical Data Center (Amante and Eakins 2009). For our purposes, the only bathymetry related variable that significantly improved model fits was the categorical variable of being on or off the shelf (OnShelf), defined as the $1000 \mathrm{~m}$ isobath (Southwell et al. 2005, 2008c; Bengtson et al. 2011). Distance from shore was also a readily available covariate but was not analyzed because it was highly correlated with distance to the sea ice edge.

In the Norwegian and South African surveys, the survey effort blocks were of variable length. Very few $<5 \%$ were longer than the $25 \mathrm{~km}$ corresponding to the resolution of the ice data raster. Furthermore, there was no information on where the seals were observed within the blocks. We therefore assigned entire blocks to the covariates obtained at the center point of the block.

\section{Analysis: modeling and predictions}

There were two steps to estimating abundances of ice seals over the broader area of the eastern Weddell Sea and waters off the coast of Queen Maud Land. First, we defined and fitted models of seal densities as a function of the environmental covariates. Second, we used the selected and fitted model to predict densities over a larger area. The South African and Norwegian data were collected in a similar manner and the methods of analysis were identical. Because the German data were unique with respect to sample sizes, range of covariates sampled, and absence of species identification, we analyzed those data separately. Finally, all prediction estimates were pooled.

\section{Norwegian and South African surveys}

In both the Norwegian and South African surveys, blocks were defined post facto based on presence of seals. Thus, every block has at least one seal (though not one of every species) and the blocks were of unequal size. The raw data were therefore unsuitable for standard approaches to modeling counts (e.g., using discrete Poisson or negative binomial generalized linear models Zuur et al. 2009). We dealt with this issue in different ways for abundant and rare seals.

Crabeater seals were present in nearly all reported blocks. We therefore modeled a log transformation of their corrected density [Number of seals $\times$ (Block Area $\times$ haulout Probability $)^{-1}$ ] as a continuous response, and explored main and interaction effects of the five covariates: DEdge, Ice, OnShelf, IceExtent and dIceExtent as explanatory factors. Because of clearly nonlinear responses to DEdge 
and Ice, we also included $\sqrt{\text { Ice }}$ and $\sqrt{\text { DEdge }}$ as candidate explanatory variables. The square root was chosen over, for example, higher-order polynomials because it has higher impact at values close to 0 (i.e., closer to the ice edge, where the distance and concentrations are low) without "exploding" at higher values for both of these variables. We weighted the densities according to the block areas for unequal sized sampling units, which ranged from $0.8 \mathrm{~km}^{2}$ to $39 \mathrm{~km}^{2}$ (median 6.5, IQR 3.4-10.7). We compared models for parsimony using Bayesian information criteria [BIC Schwarz (1978)] due to relatively few observations (162 and 64 for Norway and South Africa, respectively) relative to the number of covariates. We assessed quality of fit using $R^{2}$ between observed and model-predicted values. The log transformation resulted in adequately normal and unstructured residual distributions. The most complex model we fitted can be expressed symbolically:

$$
\begin{aligned}
Y & \sim \text { OnShelf } \times(\text { Ice }+\sqrt{\text { Ice }}) \times(\text { DEdge }+\sqrt{\text { DEdge }}) \\
& \times(\text { IceExtent }+ \text { diceExtent }) .
\end{aligned}
$$

Over half of the Weddell and leopard seal counts were 0 (A1 in Online Resource). We therefore used a binomial presence-absence model. To account for the difference in the block areas in a generalized linear modeling framework, we created a pseudo-presence-absence data set by: (1) adjusting the number of seals in each block by the appropriate time-of-day haul-out correction (figure A7 in Online Resource), (2) assigning the adjusted seal numbers to presences and absences to sub-blocks of size $0.25 \mathrm{~km}^{2}$, such that a block of area $A \mathrm{~km}^{2}$ with adjusted count $\tilde{N}_{i}$ is transformed into $\left[4 A_{i}\right]$ subblocks (where brackets denote rounding to nearest integer), to which $\tilde{N}$ are assigned pseudo-presence $=1$, and $\left[4 A_{i}-N\right]$ are assigned pseudopresence $=0$, and (3) assigning all covariates associated with the original block to the pseudo-presence table. The $0.25 \mathrm{~km}^{2}$ pseudo-block size was selected because the highest observed density after correcting for haul-out probability was around 4 ind. $\mathrm{km}^{-2}$. This reframing of the data allowed us to fit a binomial model using a similar set of covariates as for the crabeater seals.

Very few Ross seals were observed: 24 total, 10 in the Norwegian and 14 in the South African surveys. Therefore, our estimation method was simple and conservative: We divided the number of sightings by the total coverage of the respective surveys to obtain an approximate observed density estimate, multiplied this density by the average of the total ice coverage across the surveys, and divided by the average haul-out probability of $65 \%$ (Blix and Nordøy 2007). We very conservatively assumed that the densities were drawn from an exponential sampling distribution, and numerically pooled the two estimates to obtain an overall abundance.

\section{German survey}

Because any given flight for the German survey data was geographically narrow, we made the assumption that at any particular time (i.e., under a given configuration of ice densities) the fundamental response of seal densities to ice concentrations was similar across the range of the predictions. In order to produce better fitting models, it was important to have broad coverage of the different environmental covariates and as large a sample size as possible. On the other hand, ice conditions were extremely variable between years and often within a single year, with a major dynamic being the recession of the ice over the summer season (Fig. 3). Models of seal density were not expected to be consistent under these different conditions.

In order to balance the conflicting need for larger sample sizes and comparable conditions in the fitting stage, we subdivided the 37 flight days which occurred over five surveys into three groups with relatively similar ice conditions: High Ice (14 days) encompassed all of surveys 1 (1996-1997) and 5 (2000-2001), Medium Ice included the first portion of survey 2 and all of survey 4 (1999-2000), and Low Ice included the latter half of survey 2 (after January 15, 1998) and all of survey 3 (1998-1999). Over the total coastline of the study area, these three groups corresponded to total ice coverage of 40-60, 16-40 and $10-16 \times 10^{4} \mathrm{~km}^{2}$, respectively, computed as the sum of $25 \times 25 \mathrm{~km}^{2}$ SSMI grids multiplied by the ice concentrations within the grid (see Table 1 and Online Resource A for summaries of surveys and fitting model groups).

We discretized the transects into blocks of fixed area and the total number of seal groups within each block were summed to obtain a count $N_{i j}$ where $i$ and $j$ refer to the survey and the block within the flight, respectively. The lengths of the blocks were chosen such that the area of the surveyed block was $0.25 \mathrm{~km}^{2}$, i.e., ranging from 2.08 to $8.33 \mathrm{~km}$ for strip widths ranging from 120 to $30 \mathrm{~m}$. This blocking scheme led to a large number of 0 counts, but also an extreme count of 101 seals in the low ice survey in year 3 (Table A2 on Online Resource).

We used a negative binomial generalized linear model which flexibly accounts for a large number of zeros and over-dispersion (Zuur et al. 2009), using the same covariates as with the South African and Norwegian surveys: Ice, DEdge, IceExtent and dIceExtent, square-root transformations and their interactions, using BIC as a general guide for model selection. For the high and medium ice models, we added the additional constraint that the model must contain IceExtent to account for total ice cover. We modeled the number of seals that were hauled out at any given moment, and did not take the haul-out correction into 

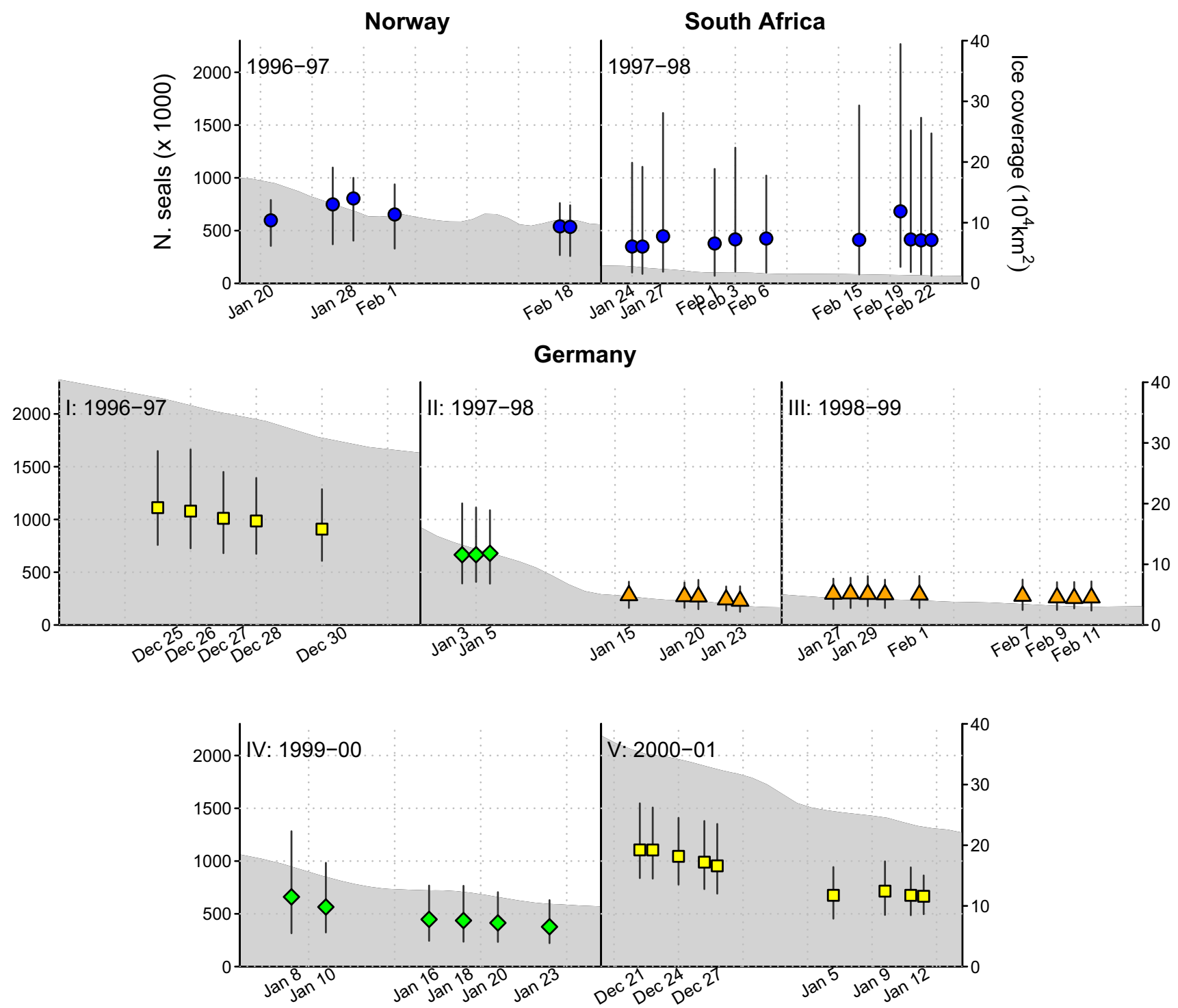

Fig. 3 Predicted abundance estimates and $95 \%$ confidence intervals for crabeater seals (upper panels) in the Norwegian (left) and South African (right) surveys, and for all seals in the five German surveys (lower panels). The gray shaded area represents the total ice coverage

account during the data fitting stage, only in the prediction stage.

\section{Obtaining abundance predictions from fitted models}

Our principal goal was to use modeled results to generate a pooled abundance estimate of all seals over a range of coastline (from $30^{\circ} \mathrm{W}$ to $10^{\circ} \mathrm{E}$ ), slightly greater than the range of of observed seals. We used the $25 \times 25 \mathrm{~km}$ grid of $\mathrm{SSM} / \mathrm{I}$ ice concentrations as our basic raster for abundance predictions. Over the range of prediction, this amounted to 2544 raster blocks $\left(1,590,000 \mathrm{~km}^{2}\right)$ of which over 1296 $\left(780,000 \mathrm{~km}^{2}\right)$ were on land. The midpoints of the blocks over the periods of the surveys (scale on right axis). In the German surveys, the three models (high ice, medium ice and low ice) predictions are presented as yellow squares, green diamonds and orange triangles, respectively

at sea were associated with SSM/I ice concentrations and distances to the $10 \%$ ice edge contour, and were determined to be within or beyond the $1000 \mathrm{~m}$ bathymetry line. Estimates for density within the blocks were obtained by applying the predicted models to the combinations of covariates in each raster.

We bootstrapped to obtain confidence intervals as follows: we resampled the observed data for each estimate with replacement, fitted the selected model, and obtained a point estimate for the resampling, repeating this procedure 1000 times for each estimate. We report the 2.5 and $97.5 \%$ quantiles of the bootstrap distribution as the $95 \%$ confidence interval. 
Table 1 Summary of seal counts and survey efforts (distance flown in $\mathrm{km}$ ) by the three survey efforts

\begin{tabular}{|c|c|c|c|c|c|c|c|c|c|c|c|}
\hline \multicolumn{3}{|c|}{ Survey } & Dates & $\begin{array}{l}\text { Distance } \\
(\mathrm{km})\end{array}$ & Flights & Crab. & Wedd. & Leop. & Ross & Unid. & Total \\
\hline \multicolumn{3}{|c|}{ Norwegian (1996-1997) } & Jan 20-Feb 18 & 1505 & 12 & 1363 & 156 & 12 & 10 & 65 & 1606 \\
\hline \multicolumn{3}{|c|}{ South African (1997-1998) } & Jan 24-Feb 22 & 1080 & 8 & 4157 & 110 & 42 & 14 & 467 & 4790 \\
\hline \multicolumn{3}{|c|}{ Germany (1996-2001) } & & 14,106 & & - & - & - & - & - & 2374 \\
\hline \multicolumn{2}{|c|}{ By season } & Subgroup & & & & & & & & & \\
\hline 1 & (1996-1997) & I & Dec 25-Dec 30 & 2841 & 5 & & & & & & 373 \\
\hline $2 \mathrm{a}$ & (1997-1998) & II & Jan 3-Jan 5 & 784 & 3 & & & & & & 51 \\
\hline $2 b$ & (1997-1998) & III & Jan 15-Jan 23 & 2446 & 5 & & & & & & 180 \\
\hline 3 & (1998-1999) & III & Jan 27-Feb 11 & 2286 & 9 & & & & & & 1063 \\
\hline 4 & $(1999-2000)$ & II & Jan $8-\operatorname{Jan} 23$ & 1282 & 6 & & & & & & 107 \\
\hline 5 & $(2000-2001)$ & I & Dec 21-Dec 27 & 4463 & 9 & & & & & & 600 \\
\hline
\end{tabular}

The German survey, which did not identify species, included efforts over five winter seasons which we broke into three subgroups of high, medium and low ice (I, II and III) for modeling

A model from an entire survey was extrapolated to predict densities over a single day. Thus one model was separately used to predict the number of seals for each flight day. Because individual surveys did not always identify many seals, we expected the confidence intervals to be relatively large. However, under the assumption that the total number of seals over the entire region remains relatively constant, we could average the point estimates of the different days within a survey, and obtain confidence intervals that are narrower than the confidence intervals around a single day's estimate (Ver Hoef et al. 2014). We could not properly consider the predictions derived from within a given year to be independent, since the models were parameterized with all the data collected in a given year. But across the three ice models in the German survey and the South African and Norwegian surveys, the estimates were clearly independent and we could pool the predictions using a Monte Carlo (MC) simulation to obtain confidence intervals for the overall abundance of the seals in the area as follows:

To do this, we first fitted a gamma distribution to the mean and 2.5 and $97.5 \%$ confidence interval of the grid cell specific predictions. We then drew estimates from those gamma distributions $10^{5}$ times. For each MC draw, we obtained a single point estimate for each of the three German models and one for each of the Norway and South Africa species, and report the overall mean and 2.5 and $97.5 \%$ quantile as the confidence interval. Symbolically:

$N_{m c, j}=\left(\widehat{N}_{g e r 1, j}+\widehat{N}_{g e r 2, j}+\widehat{N}_{g e r 3, j}+\widehat{N}_{n o r, j}+\widehat{N}_{s a, j}\right) / 5$

$\widehat{N}=\overline{N_{m c}}\left(C I=Q_{0.25}\left[N_{m c}\right], Q_{0.975}\left[N_{m c}\right]\right)$,

where $j$ represents the Monte Carlo draw, $\widehat{N}_{i, j}$ represents draws from the respective distributions for each model (the three German, Norwegian and South African models, respectively) and $Q$ refers to the quantile of the MC distribution.

An additional challenge was obtaining species-specific estimates from the German data, where no species were identified. The South African and Norwegian surveys overlapped with much of the same area as the German survey (longitude: $45.08^{\circ} \mathrm{W}-8.16^{\circ} \mathrm{W}$, latitude: $73.8^{\circ} \mathrm{S}-$ $62.4^{\circ}$ ). We took the proportion of total observations of the other seals by species (of 8044 seals, $94.1 \%$ crabeaters, $4.5 \%$ Weddell seals, $1 \%$ leopard seals, $0.4 \%$ Ross seals) to be representative of the seal abundances within the German survey area and numerically drew an individual species identification from a multinomial distribution with these probabilities. We used this technique mainly to obtain an overall crabeater seal estimate; for the remaining seals we report only the predictions from the direct observations of the South African and Norwegian surveys.

For all analyses we used the $\mathrm{R}$ statistical software package (R Development Core Team 2016), including the "MASS" package (Venables and Ripley 2002) for generalized linear modeling and the "maps" and "mapdata" (Becker et al. 2013) packages for mapping.

\section{Results}

\section{Norwegian and South African surveys}

The Norwegian effort consisted of a total of eight surveys flown on six days between January 20 and February 18 in 1997 (Table A1, Fig. 1, see also Online Resource A1 for more details). The total distance flown was $1505 \mathrm{~km}$. The ice coverage was relatively high at the beginning of the survey, with a mean ice extent (distance from shore to ice edge) of $253.4 \mathrm{~km}$ during the first survey dropping to 80.0 
$\mathrm{km}$ by the last survey. A total of 1606 seals were sighted in 179 sightings. Of the sighted seals, 1363 (84.9\%) were crabeater seals, $156(9.7 \%)$ were Weddell seals, 12 and 10 $(<1 \%)$ individual leopard and Ross seals, respectively (Fig. 4), and 65 (4\%) were unidentified. We ignored the unidentified seals in subsequent analyses. Seal group sizes ranged from 1 to a maximum of 48 for the crabeater seals and 56 for the Weddell seals. The overall median groups size was 4 (IQR: 1-9) for all species and 7 (IQR: $3-11)$ for crabeater seals only. Only 43 seals $(2.8 \%)$ of the identified species were sighted individually. In total, 841 $(62 \%)$ of the crabeater seals and $110(70.5 \%)$ of the Weddell seals were found in groups of size 10 or greater. Each sighting of seals was reported within a single block; the blocks were of various lengths, which were converted to areas using the effective strip width described above. The areas of the 179 blocks ranged from 0.89 to $38.9 \mathrm{~km}^{2}$ (mean 8.26, sd 6.48).

The South African survey consisted of a total of 12 surveys flown on 11 days between January 24 and February 22 in 1998 (Table 1 and Online Resource Table A1). All transects in the South African survey were flown along the narrow shelf of the region with no locations deeper than $3000 \mathrm{~m}$ (Fig. 1). The total distance flown was $1080 \mathrm{~km}$. The surveyed ice concentrations were low ranging from 0 to $44 \%$, compared to $26-100 \%$ in the preceding year's Norwegian survey. Consequently, the distances to the ice edge and shore of any sighted seal group was relatively short, between 0 and $54 \mathrm{~km}$. In total, 4790 seals were sighted in 188 groups. Of these, $110(9.75 \%)$ were unidentified, and therefore excluded from the analysis. Of the remaining, $4157(96.2 \%)$ were crabeater seals, 151
$(2.5 \%)$ were Weddell seals, $42(1 \%)$ were leopard seals and $14(0.3 \%)$ were Ross seals (Fig. 4). Note that 2515 crabeater seals were seen on one single survey (\#11: Feb 20,1998 ) accounting for over $50 \%$ of all observed seals. This survey included six groups of seals over 100 and up to 899 individuals, contrasted with a median group size of 6 (IQR: 2-16) for all species of seals and 24 (IQR: 8.75-51.1) for just crabeater seals. See also Bester and Odendaal $(1999,2000)$ for additional details and summarized results of the South African survey.

\section{Crabeater seals}

We modeled the density of crabeater seals by fitting 21 models, denoted M0 for the null model to M20 for the most complex model (Eq. 3). For the Norwegian data, we selected M4, which included the IceExtent and dIceExtent covariates in interaction with the OnShelf variable:

$Y \sim$ OnShelf $\times($ IceExtent + dIceExtent $)$.

where $Y$ is the $\log$ of the density of seals. This relatively simple model had the lowest BIC of the models that included the OnShelf covariate. Diagnostic tests confirmed that normality and homoskedasticity assumptions were satisfied. The coefficient values and significance of effects are presented in Table 2. Seal density, predictably, was lower when ice extent was greater, but increased when sea ice receded more rapidly. Densities also tended to be higher on the shelf and on more concentrated ice. Interestingly, the two-way interactions with OnShelf were significant. For example, the IceExtent $\times$ OnShelf interaction was high, negative and significant, indicating that under
Fig. 4 Estimated densities of observed seals of all four seal species (four labeled panels) along the survey transects for the Norwegian (red dots) and South African (yellow dots) surveys. Dot sizes reflect the haul-out corrected densities in the observation blocks (see legends for scale). The pale pink and yellow lines represent the transect flights of the Norwegian and South African surveys, respectively
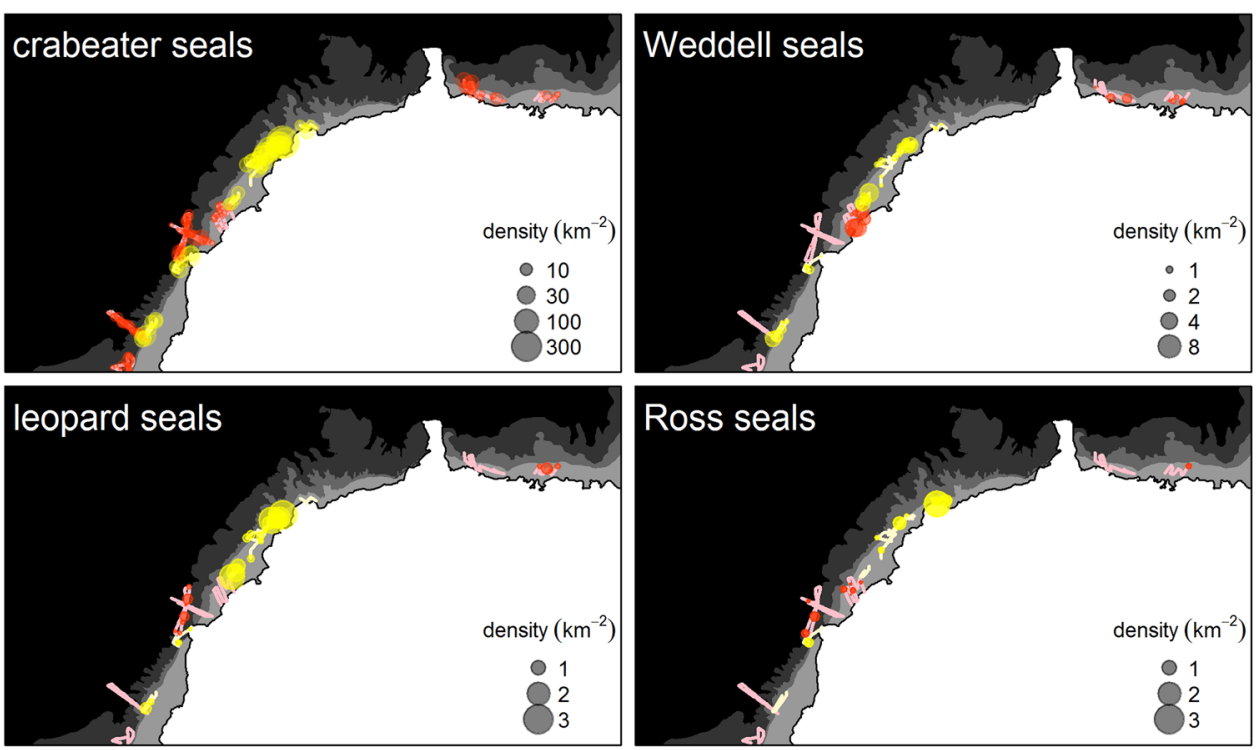

Norwegian survey

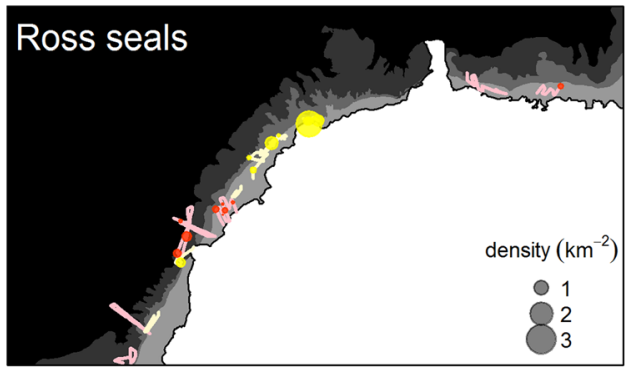

South African survey 
Table 2 Coefficients and significance of crabeater linear models on log density (Norwegian and South African surveys), and the three negative binomial models for the German data

\begin{tabular}{|c|c|c|c|c|c|}
\hline \multirow{3}{*}{$\begin{array}{l}\text { Factor } \\
\text { OnShelf }\end{array}$} & \multirow{3}{*}{$\begin{array}{l}\text { Norway } \\
\text { (Crabeater } \\
-1.72 * * *\end{array}$} & \multirow{3}{*}{$\begin{array}{l}\text { South Africa } \\
\text { als }) \\
-0.85^{*}\end{array}$} & \multicolumn{3}{|c|}{ Germany (all seals) } \\
\hline & & & \multirow[t]{2}{*}{ High ice } & \multirow{2}{*}{ Medium ice } & \multirow{2}{*}{$\frac{\text { Low ice }}{-1.5319^{* * *}}$} \\
\hline & & & & & \\
\hline DEdge & & & $-1.30 * *$ & $-0.38 * *$ & \\
\hline$\sqrt{\text { DEdge }}$ & & & $-0.85^{*}$ & & \\
\hline IceExtent & $-0.43^{* * *}$ & $-0.86^{* *}$ & $-0.40 * *$ & 0.104 & \\
\hline dIceExtent & $-0.30 * * *$ & & $-0.21 * *$ & & \\
\hline Ice & & & 0.50 & & \\
\hline$\sqrt{\text { Ice }}$ & & & -0.25 & $-0.585 * * *$ & \\
\hline DEdge:IceExtent & & & $1.17 * * *$ & $* *$ & \\
\hline dIceExtent:Ice & & & $-0.95^{* *}$ & & \\
\hline dIceExtent: $\sqrt{\text { Ice }}$ & & & $-1.22 * * *$ & $-0.40 * *$ & \\
\hline OnShelf:IceExtent & $-1.36 * *$ & 0.78 & & & \\
\hline OnShelf:dIceExtent & $-1.99 * * *$ & & & & \\
\hline$R^{2}$ & 0.317 & 0.17 & & & \\
\hline$\widehat{\theta}$ & & & $0.78(0.07)$ & $0.53(0.15)$ & $0.055(5 e-3)$ \\
\hline$\Delta \mathrm{BIC}$ & 36.2 & 6 & 38.3 & 9.06 & 1.6 \\
\hline
\end{tabular}

All parameter estimates are given for standardized covariates (except the binomial OnShelf variable)

The significances are coded according to $p$-value: $* * *<0.001 \leq * *<0.01 \leq *<0.05 \leq-<0.10$ conditions of more ice, there was a stronger relative preference for being off the continental shelf.

The South African data were collected late in a season with exceptionally low ice. The selected model that provided both low BIC values and stable predictions contained main and interaction effects for being on the shelf and ice extent (coefficients and significance in Table 2). As in the Norwegian model, densities were higher at lower ice extents and lower on the continental shelf.

We used these models to estimate the number of crabeater seals in the region between $30^{\circ} \mathrm{W}$ and $10^{\circ} \mathrm{E}$. While all of the data were used to parameterize the models for each national survey, the corresponding models were used to predict total regional abundances separately for each day of the survey (Fig. 3 upper panels, Table 2), as the ice covariates all varied from day to day. The point estimate and bootstrapped $95 \%$ confidence intervals are presented in Table 2 and Fig. 3. The final point estimates from each of these surveys were rather consistent: 601,700 $(327,900-980,450)$ and 451,600 (24,900-1,490,300), though the confidence intervals of the South African estimates were much wider. With the assumption that the number of seals was constant within error across years, we obtained an overall pooled estimate by drawing point estimates from a gamma distribution fitted to the mean and bootstrapped $95 \%$ confidence intervals, and averaging the estimates (one from each survey) together with the results of the German survey (see Table 4 and results below).
Table 3 Coefficients and significance of binomial glm's for the two Weddell seal and leopard seal models

\begin{tabular}{|c|c|c|c|c|}
\hline \multirow[b]{2}{*}{ Factor } & \multicolumn{2}{|c|}{ Weddell seals } & \multicolumn{2}{|c|}{ Leopard seals } \\
\hline & Norway & South Africa & Norway & South Africa \\
\hline OnShelf & & $1.09 * * *$ & $-6.95^{-}$ & $0.88 * * *$ \\
\hline DEdge & 2.19 & $3.25^{* * *}$ & & \\
\hline$\sqrt{\text { DEdge }}$ & $0.20 * * *$ & $-2.77 * * *$ & & \\
\hline IceExtent & $-11.7 * * *$ & $-0.15^{* *}$ & & $-0.62 * * *$ \\
\hline Ice & & & $-0.88 * * *^{-}$ & \\
\hline DEdge:IceExtent & $-26.8 * * *$ & $-1.14 * * *$ & & \\
\hline$\sqrt{\text { DEdge: IceExtent }}$ & $16.0 * * *$ & & & \\
\hline OnShelf:Ice & & & $-5.01 * * *$ & \\
\hline
\end{tabular}

All parameter estimates are given for standardized values of the covariates

The significance is coded as in Table 2 


\section{Weddell seals}

There were 156 Weddell seals in total observed in the Norwegian surveys (Table A1). All but three observations occurred on the continental shelf, despite over $70 \%$ of the effort occurring off the continental shelf (Fig. 4). This reflected the strong shallow water preference of the Weddell seals, motivating us to constrain our predictions for the fitted model only to the shelf area. After accounting for haul-out probability (figure A7) and distributing the adjusted number of seals onto $0.25 \mathrm{~km}^{2}$ blocks, there were a total of 240 pseudo-presences on 2265 on-shelf blocks.

Visual exploration of the relationship to ice concentration and distance to ice edge suggested the inclusion of higher-order (square root) terms. The model we selected, based on prioritizing highly significant covariates and dropping nonsignificant terms, excluded ice concentration covariates entirely (Table 3) and can be expressed:

$N \sim($ DEdge $+\sqrt{\text { DEdge }}) \times$ IceExtent.

In the South African surveys 110 Weddell seals were sighted (Table A1). Seals were found both on and off the shelf (Fig. 4). After correcting for haul-out probability and allocating among blocks, there were 168 pseudo-seals on 1994 blocks. The smaller number of blocks compared to the Norwegian survey (despite including both on and offshelf areas) is explained by the much lower quantity of ice in 1998 and consequently shorter transects (Fig. 1). The selected model contained the same main effect as in the Norwegian model, with the inclusion of presence on shelf and one fewer two-way interaction (Table 3):

$N \sim$ DEdge $+\sqrt{\text { DEdge }} \times$ IceExtent + OnShelf

All included effects were significant.

The overall point estimates for Weddell seals were largely consistent across the dates of the surveys and across both years (Fig. 5; Table A7), despite the large difference in ice conditions between and within the two years. The point estimates varied between about 29,000 and 45,000, with coefficients of variation around $10 \%$ for each estimate. We considered the Norwegian and South African data to be independent estimates and numerically pooled them by averaging a randomly drawn point estimate from the bootstrap distributions from both countries, averaging the two results, repeating the procedure 10,000 times and reporting the median and $95 \%$ range of the means. This

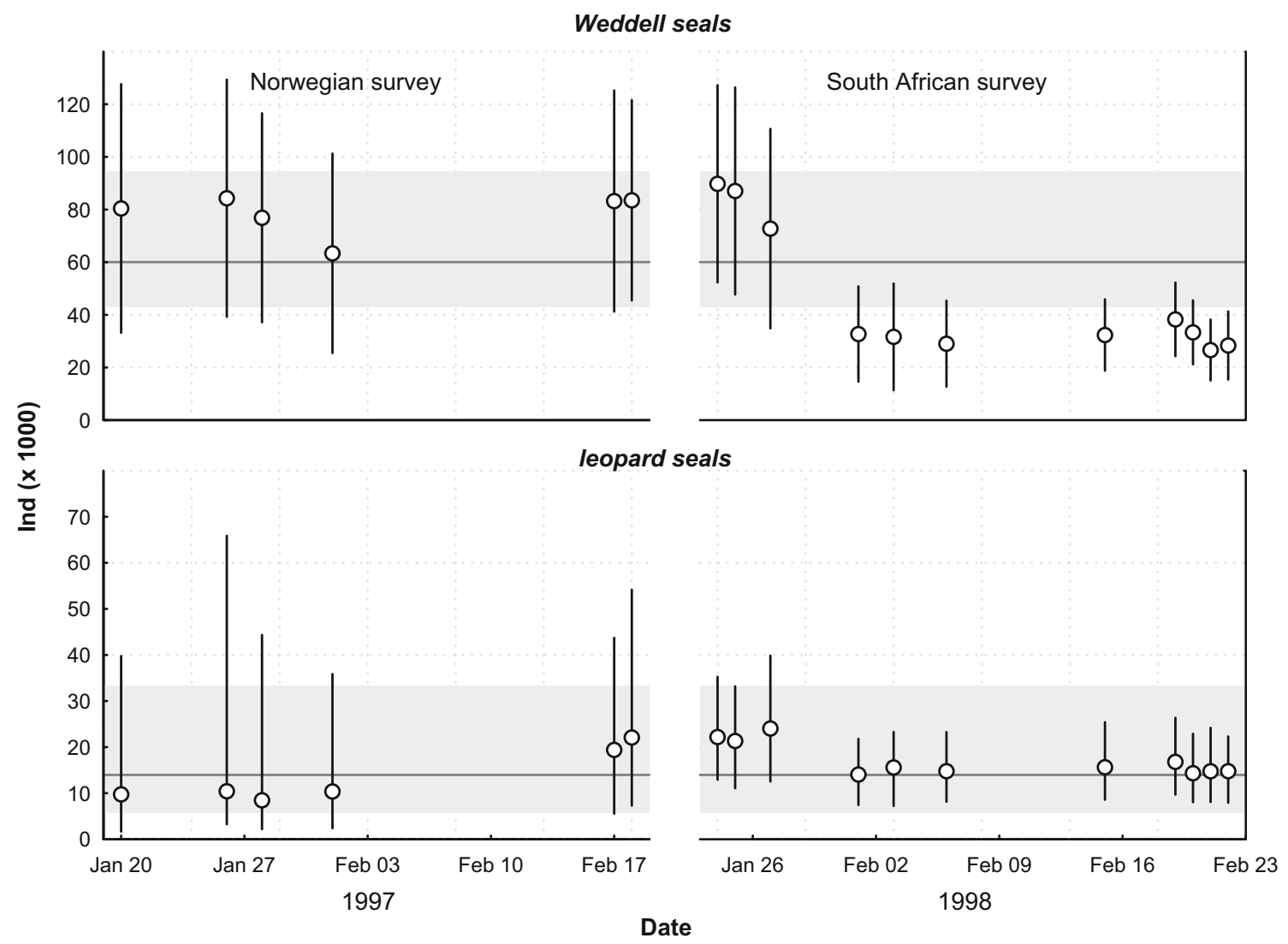

Fig. 5 Weddell seal (upper panels) and leopard seal (lower panels) abundance estimates and $95 \%$ confidence intervals for each of the Norwegian (left panels) and South African (right panels) surveys. The

horizontal line and shaded region represent the overall pooled estimate and $95 \%$ confidence intervals based on numerically sampling from bootstrap distributions of the two surveys 
procedure yielded an aggregated point estimate of 59,990 (95\% CI 43 170-94 390).

\section{Leopard seals}

There were 12 (11 sightings) and 42 (23 sightings) leopard seals observed in the Norwegian and South African surveys, respectively (Fig. 4, Table A1). For both Norwegian and South African surveys, we fitted binomial GLMs with main effects of ice concentration, mean extent of ice and distance to ice edge with potential interactions with presence on shelf and used both Akaike's (AIC) and Bayesian Information Criterion (BIC) to help guide model selection.

In the Norwegian survey, the selected model included ice concentration and presence on shelf covariates (Table 3) with all effects significant, and the lowest AIC values of several models (Table A13). Densities tended to be higher on the shelf and lower with higher ice concentration. In the South African survey, the model with lowest AIC (and BIC) contained mean ice extent and presence on shelf main effects (Table 3). The effect of being on the shelf was positive and highly significant, though weaker than in the Norwegian survey, and probability of presence was significantly higher at smaller ice extents.

Bootstrapped abundance estimates using the Norwegian model yielded six day-by-day total abundance estimates ranging from $3380(500-10,400)$ leopard seals to 8840 (2700-24,330) individuals (Table A7), while the South African model yielded 11 day-by-day estimates of abundance ranging from $5620(2970-8720)$ to 9620 (5010-15,900) individual seals, prior to applying the haulout correction (Table A7). When adjusted for haul-out probability (using the 0.4 factor) and jointly pooling the estimates using the methods used for crabeater and Weddell seals, we obtained an overall abundance estimate of 13,990 (95 \% CI 5700-33,600) leopard seals in the region (Fig. 5).

\section{Ross seals}

There were 10 Ross seals observed over $1478 \mathrm{~km}^{2}$ in the Norwegian data, yielding an average density of $6.7 \times 10^{-3} \mathrm{~km}^{-2}$. Multiplied over an average of 123,000 $\mathrm{km}^{2}$ of ice coverage, this yielded an estimated abundance of 832 seals, or 1281 (32-4724) after correcting for haulout probability (Table 4).

In the South African survey, 14 individuals were observed over $499.5 \mathrm{~km}^{2}$ of ice, yielding a much higher average density of $2.8 \times 10^{-2}$. Extrapolated over a much smaller average ice area of $16,910 \mathrm{~km}^{2}$, the resulting point estimate after correcting for haul-out probability was 729 (18-2690) individuals. Pooling these two results yielded an
Table 4 Total abundance estimates by species for the surveys $\left(\times 10^{3}\right.$ ind. for all except Ross seals)

\begin{tabular}{lll}
\hline Survey & Estimate & $(95 \% \mathrm{CI})$ \\
\hline Crabeater seal & $\times 1000$ ind. & \\
German survey: Low Ice & 245 & $(155-363)$ \\
German survey: Medium Ice & 409 & $(139-944)$ \\
German survey: High Ice & 824 & $(423-1398)$ \\
Norwegian survey & 584 & $(328-963)$ \\
South African survey & 574 & $(65-2158)$ \\
Pooled & 514 & $(337-886)$ \\
Weddell seals & & \\
Norwegian survey & 82.4 & $(55.3-109.3)$ \\
South African survey & 33.6 & $(20.8-99.6)$ \\
Pooled & 60.0 & $(43.2-94.4)$ \\
Leopard seals & & \\
Norwegian survey & 7.8 & $(2.54-60.4)$ \\
South African survey & 16.5 & $(8.5-31.8)$ \\
Pooled & 13.2 & $(5.50-39.7)$ \\
Ross seals & ind. & \\
Norwegian survey & 1280 & $(32-4720)$ \\
South African survey & 729 & $(18-2690)$ \\
Pooled & 828 & $(119-2894)$ \\
\hline For each & &
\end{tabular}

For each species, we considered estimates from unique surveys and or model groupings (for the German data) to be independent estimates and pooled these to produce the final number. For the German seals, we corrected the total estimated abundance of seals by the proportion of crabeater seals seen in the Norwegian and South African surveys ( $94.1 \%$ of total). For more detailed abundance estimates, see Online Resource C

abundance estimate of 829 (117-2892) Ross seals in our study region.

\section{German surveys}

In the German surveys, 1158 seal sightings were made of 2376 individual seals. Group sizes varied, although the majority of the sightings were of single seals (Online Resource Table A2). All groups larger than 10 seals (including up to 101 seals) occurred in survey 3 (1998-1999), which had the lowest amount of ice of any other year, in the inlets near the shore of the westernmost part of the surveyed region (Fig. 1). The observed densities of seals on ice ranged from a mean of 1.30 (s.d. 0.49 ) $\mathrm{km}^{-2}$ in the highest ice year (1996-1997) to $4.04(0.55) \mathrm{km}^{-2}$ in the lowest ice year (1998-1999).

We summarize the details of the three (high ice, medium ice, low ice) fitted models in Table 2, visualize predicted densities over several days of the survey in Online Resource A8, and provide additional detail of the model selection in Online Resource D.

The selected high ice model excluded the shelf covariate, but included all the ice-related main effects, including 
ice concentration and square root of ice concentration, distance to ice edge and square root of distance to ice edge, and four interactions, nearly all highly significant, with a small amount of over-dispersion $(\widehat{\theta}=0.78$ (s.d. 0.07) Table 2). Seals were found at higher densities on higher ice concentrations and closer to the ice edge, with overall densities increasing with less overall ice extent, and the effect of distance from the edge increasing with more rapidly receding ice, reflecting the increased concentration of seals at the ice edge. The selected medium ice model included somewhat fewer terms (three main effects and one interaction) and higher over-dispersion $(\widehat{\theta}=0.53(0.15))$, due mainly to fewer data available (158 seals on 514 blocks compared to 950 seals over 2371 blocks for the high ice model). The model suggested similar patterns of lower density further from the ice edge and higher concentrations at the edge with more quickly receding ice. The low ice model predicted the highest density of seals over the smallest amount of ice (1295 individuals over 1243 blocks), and the only significant covariate was presence on the shelf, where seal density was predicted to be five times higher than off the shelf (4.4 and $0.95 \mathrm{~km}^{-1}$, respectively). Predictions of these three models for three different days are presented in figure $\mathrm{A} 8$.

Using the sequence of models to predict total abundance (of all ice seal species) over the study range on the survey dates, provided a range of point estimates between 227,000 and 1,112,200 (Table A5; Fig. 3) in the study area. The lowest estimates tended to be for low ice models and the highest estimates were for the high ice models, but the low ice confidence intervals overlapped with the medium ice intervals, and the medium ice intervals with the high ice intervals; in total $68.5 \%$ of all pairs of estimates had overlapping confidence intervals.

Pooling all of the German results, using each of the three model estimates as independent samples (and assuming a constant population across years) yielded an overall abundance of 525,000 seals (95\% CI 332,660-791,700). Adjusting for the empirical ratios of crabeater seals against other seals in the other two surveys and numerically pooling these estimates with the Norwegian and South African estimates yields a final crabeater seal abundance estimate for our study area of 515,000 $(337,000-887,000)$ (Table A5).

\section{Discussion}

We estimated the density and distribution of ice seals over a $40^{\circ}$ longitude span portion of the Southern Ocean $\left(30^{\circ} \mathrm{W}-10^{\circ} \mathrm{E}\right)$, slightly larger than the extent of the multinational survey effort. Together with the areas of Antarctica surveyed by APIS participants from Australia $\left[64^{\circ} \mathrm{E}-150^{\circ} \mathrm{E}\right.$, Southwell et al. $\left.(2005,2008 \mathrm{a}, \mathrm{b}, \mathrm{c})\right]$, the United States $\left[150^{\circ} \mathrm{E}-100^{\circ} \mathrm{W}\right.$, Bengtson et al. (2011)] and United Kingdom $\left[90^{\circ} \mathrm{W}-30^{\circ} \mathrm{W}\right.$, Forcada et al. (2012)] (Table 5), the survey results presented here complement and complete a near-circumpolar study of ice seal distribution and abundances. Importantly, all of these survey estimates similarly incorporate detection at distance, corrections for haul-out probability, and models of density based on environmental covariates.

\section{Abundance and densities}

In earlier studies on the distribution and abundance of pack ice seals conducted in the eastern Weddell Sea (e.g., Erickson and Hanson 1990) during late summer (as in the present study), seals were counted in a narrow strip on either side of a ship (Condy 1976, 1977) or aircraft (Bester et al. 1995; Bester and Odendaal 2000; Bester et al. 2002), and time-corrected for maximal haulout (Erickson et al. 1989) where appropriate. Those studies are therefore not directly comparable to the present study, although Bester and Odendaal (2000) treated the raw data on which the present paper is based in the same way (strip survey not accounting for probability for detection at distance) to compare with those studies mentioned above [Table 2,
Table 5 Abundance estimation of crabeater and Weddell seals across Antarctic surveys

\begin{tabular}{|c|c|c|c|c|c|}
\hline Range & Year & APIS partner & \multicolumn{2}{|c|}{ Estimate $(\mathrm{CI})\left(\times 10^{3}\right.$ ind. $)$} & Reference \\
\hline \multicolumn{6}{|l|}{ Crabeater seals } \\
\hline 1. $30^{\circ} \mathrm{W}-10^{\circ} \mathrm{E}$ & 1996-2000 & No., S.A., Ger. & 514 & $(337-886)$ & This study \\
\hline 2. $\quad 64^{\circ} \mathrm{E}-150^{\circ} \mathrm{E}$ & $1999 / 2000$ & Aus. & 946 & $(726-1396)$ & Southwell et al. (2008a) \\
\hline 3. $150^{\circ} \mathrm{E}-100^{\circ} \mathrm{W}$ & $1999 / 2000$ & USA & 1736 & $(1219-2472)$ & Bengtson et al. (2011) \\
\hline 4. $90^{\circ} \mathrm{W}-30^{\circ} \mathrm{W}$ & 1998/1999 & UK & 3042 & $(2530-3703)$ & Forcada et al. (2012) \\
\hline \multicolumn{6}{|l|}{ Weddell seals } \\
\hline 1. $30^{\circ} \mathrm{W}-10^{\circ} \mathrm{E}$ & 1996-2000 & No., S.A., Ger. & 60 & $(43-94)$ & This study \\
\hline 2. $\quad 64^{\circ} \mathrm{E}-150^{\circ} \mathrm{E}$ & $1999 / 2000$ & - & - & - & \\
\hline 3. $150^{\circ} \mathrm{E}-100^{\circ} \mathrm{W}$ & $1999 / 2000$ & USA & 331 & $(144-759)$ & Bengtson et al. (2011) \\
\hline 4. $90^{\circ} \mathrm{W}-30^{\circ} \mathrm{W}$ & 1998/1999 & UK & 369 & $(245-531)$ & Forcada et al. (2012) \\
\hline
\end{tabular}


Bester and Odendaal (2000)]. Erickson and Hanson (1990) did report an estimated abundance of crabeater seals around 806,000 for the eastern Weddell sea (between $20^{\circ} \mathrm{W}$ and $10^{\circ} \mathrm{E}$ in their definition), which falls within the range of our estimates.

Despite the different approaches, the South African survey (this study; Bester and Odendaal 2000), returned similar results in the proportions of different species observed (crabeater seals 96.0-95.4\%; Weddell seals 2.9-2.5 \%; Ross seals $<1 \%$; leopard seals $0.3-0.5 \%$ ). The Norwegian census delivered a higher proportion of Weddell seals $(9.7 \%)$ at the cost of crabeater seals $(84.9 \%)$. This difference is likely explained by the fact that the Norwegian team surveyed more fast ice, where Weddell seals are more commonly found, than the South African survey, which concentrated on pack ice. In terms of seal density, the surveyed area in the eastern Weddell Sea was largely devoid of pack ice, while a well circumscribed pack ice field remained in the western Weddell Sea during the 1998 survey. At a mean density of $21.16 \mathrm{nmi}^{-2}\left(6.17 \mathrm{~km}^{-2}\right)$ over an area of $244.2 \mathrm{nmi}^{2}\left(837.6 \mathrm{~km}^{2}\right)$, these were the highest densities on record for crabeater seals with up to $411.7 \mathrm{nmi}^{-2}\left(120 \mathrm{~km}^{-2}\right)$ being found in small areas according to unadjusted counts by Bester and Odendaal (2000). The overall high densities of seals $\left(30.18 \mathrm{nmi}^{-2}\right.$ $\left.\left[8.8 \mathrm{~km}^{-2}\right]\right)$ recorded for the eastern Weddell Sea (27.46, 0.27 and $0.66 \mathrm{nmi}^{-2}$ [8.0, 0.08, $0.19 \mathrm{~km}^{-2}$ ] for crabeater, leopard and Weddell seals, respectively) was a consequence of the drastically reduced ice cover (Bester and Odendaal 2000) and the inverse relationship between cover and seal densities (Eklund and Atwood 1962; Erickson et al. 1973; Bester et al. 1995. Ross seal densities $\left(0.08 \mathrm{nmi}^{-2}\left[0.02 \mathrm{~km}^{-2}\right]\right)$ were the lowest on record for the area, although this was consistent with the decrease in abundance of the species from east to west previously reported in the Weddell Sea (Erickson et al. 1973; Condy 1977).

\section{Environmental covariates}

There are considerable challenges to modeling the distributions and densities of Antarctic ice seals. On a local scale, densities depend on the presence and distribution of ice, which varies greatly both within and across seasons, and on ecological constraints of feeding and breeding, which occur during the summer season when these surveys were undertaken. The relationships with ice covariates are complex: densities are highest when there is the least ice, higher ice concentrations are generally preferred, but too much ice appears to have an inhibitory effect, perhaps reflecting a constraint on movement, maintenance of foraging groups, or access to open water. The shelf may be preferred, but in heavy ice years may be less accessible, especially in a region like Queen Maud Land where the shelf is narrower than elsewhere. Furthermore, the surveys themselves were performed under variable conditions and using rather different survey protocols and instruments.

Nonetheless, by pooling these surveys, using various modeling techniques (e.g., discrete models of counts for the uniform block-size German data compared to transformed linear models of densities for the variable-blocksize South African and Norwegian data) and grouping observations over similar conditions, we were able to fit models and make predictions that yielded broadly consistent results across surveys. The selected habitat covariate models reflected the complexity of the seal response to ice. In high ice years (e.g., the Norwegian survey and large portions of the German survey) significant higher-order terms for ice concentration and distance to ice edge reflected the intermediate preferences of the seals. The inclusion of the total ice extent and change in ice extent covariates allowed us to take the dynamic nature of the ice cover into account.

Under the naive assumption that haul-out behavior remained consistent and long-distance displacements were minimal, we would anticipated that accounting for total amount and fluxes of ice in our model would lead to stable estimates, regardless of the amount of ice. In fact, in all cases we found fewer seals in areas-and seasons-with less ice, consistent with the idea that seals move, at times over large distances, to remain on the pack ice (Bester and Odendaal 1999). In 1998, an extremely low ice year associated with a strong El Niño/ Southern Oscillation (ENSO) event, local densities and aggregations of seals were many orders of magnitude higher than in other years. Because the range of covariates was considerably narrower, the models were simpler, indicating significant preferences of both crabeater and, especially, Weddell seals for being over the continental shelf (Fig. 4). It is interesting to note that the aggregations were rather localized, i.e., large aggregations in one area were adjacent to apparently similar areas of pack ice with no seals (Bester and Odendaal 1999, 2000). The patchiness of the distribution suggests either some social interactions or historical contingency to the large-scale distributions, possibly associated with ENSO associated local fluctuations in krill densities and distribution. On the other hand, it is possible that low pack ice conditions also influence the haul-out behavior of seals, or the detectability of individuals or groups in ways that systematically bias the abundance estimate downward. The question of behavioral responses to low ice conditions is an important one to explore further, not only for its potential impact on abundance estimates, but because low ice conditions are increasingly likely (Turner et al. 2009). 


\section{Comparison to other parts of Antarctica}

Importantly, the ability to pool the results across years and across completely different survey techniques allowed us to narrow the precision of the prediction to a coefficient of variation on par with the more consistent and geographically broader Australian and US surveys. The longitudinal density of crabeater seals (i.e., number of individuals per degree longitude) in our study region was similar to numbers reported for other parts of Antarctica by APIS Program participants, both in magnitude and precision (Table 5). Crabeater seal abundance in our study was $14,315(10,650-19,175)$ over $40^{\circ}$, very close the 13,520 $(10,377-19,981)$ reported by Southwell et al. (2008a) over $70^{\circ}$ and $15,781(11,081-22,473)$ over $110^{\circ}$ reported by Bengtson et al. (2011). Forcada et al. (2012) reported on abundance and density estimations of a survey conducted by APIS partners from the UK in 1999 along the western coast of the Antarctic peninsula and western portion of the Weddell Sea (between $90^{\circ}$ and $30^{\circ} \mathrm{W}$ ). The estimate in that region (ca 3 million crabeaters) leads to a much greater per-longitude density, but that is readily explained by the much longer per-longitude coastline length along the Antarctic Peninsula, which has a generally North-South orientation.

A comprehensive unification of the APIS program estimates-an immediate future goal—will provide the most precise global estimate of the abundance of all Antarctic seal species to date. This will serve as an essential reference point for monitoring changes in abundance of these species and related marine ecosystems, even as the climate in the Antarctic undergoes rapid change (Turner et al. 2009, 2015).

\begin{abstract}
Acknowledgments The authors would like to thank Jeff Laake for invaluable comments on the data analysis, Monica Sundset for assistance in the field and Jay VerHoef for the Weddell seal haul-out correction. The research was supported in part by the Norwegian Research Council and the Norwegian Polar Research Institute (NARE 1996/97). The Alfred Wegener Institute provided generous helicopter support and berths on board the RV Polarstern during the EASIZ II cruise under the leadership of Wolf Arntz, as well as aircraft capacity during the EMAGE campaigns. The University of Pretoria, the South African National Research Foundation (then the Foundation for Research Development) and the Department of Environmental Affairs variously provided financial and logistical support for the South African contingent. The Hanse-Wissenschaftskolleg in Delmenhorst, Germany, kindly provided a Fellowship to MNB during the final write-up of this paper.
\end{abstract}

Open Access This article is distributed under the terms of the Creative Commons Attribution 4.0 International License (http://crea tivecommons.org/licenses/by/4.0/), which permits unrestricted use, distribution, and reproduction in any medium, provided you give appropriate credit to the original author(s) and the source, provide a link to the Creative Commons license, and indicate if changes were made.

\section{References}

Amante C, Eakins BW (2009) ETOPO1 1 Arc-Minute Global Relief Model: Procedures, Data Sources and Analysis. NOAA Technical Memorandum NESDIS NGDC-24, National Snow and Ice Data Center

Becker RA, Wilks AR, Brownrigg R, Minka TP (2013) Maps: Draw Geographical Maps. http://CRAN.R-project.org/package=maps

Bengtson J, Laake J, Boveng P, Cameron M, Hanson M, Stewart B (2011) Distribution, density, and abundance of pack-ice seals in the Amundsen and Ross seas, Antarctica. Deep-Sea Res Pt II 58:1261-1276

Bester M, Erickson A, Ferguson J (1995) Seasonal change in the distribution and density of seals in the pack ice off Princess Martha Coast, Antarctica. Antarct Sci 7:357-364

Bester M, Ferguson J, Jonker F (2002) Population densities of packice seals in the Lazarev Sea, Antarctica. Antarct Sci 14:123-127

Bester M, Odendaal P (1999) Abundance and distribution of Antarctic pack ice seals in the Weddell Sea. In: Arntz W, Gutt J (eds) The Expedition ANTARKTIS XV/3 (EASIZ II) of Polarstern in 1998, vol 301. Alfred-Wegener-Institut für Polar- und Meeresforschung. Berichte zur Polarforschung, Bremerhaven, pp 102-107

Bester M, Odendaal P (2000) Abundance and distribution of Antarctic pack ice seals in the Weddell Sea. In: Davison W, HowardWilliams C, Broady P (eds) Antarctic ecosystems: models for wider ecological understanding. Caxton Press, Christchurch, pp 59-63

Bester M, Odendaal P, Gurarie E (2016) Seal census primary data during Polarstern expedition ANT-XV/3 (PS48), vol 301. Alfred-Wegener-Institut für Polar- und Meeresforschung. Berichte zur Polarforschung, Bremerhaven. doi:10.1594/PAN GAEA.861938

Blix A, Nordøy P (2007) Ross seal (Ommatophoca rossii) annual distribution, diving behaviour, breeding and moulting, off Queen Maud Land, Antarctica. Polar Biol 30:1449-1458

Comiso J (1990) DMSP SSM/I Daily and Monthly Polar Gridded Bootstrap Sea Ice Concentrations (updated quarterly, dates used: Feb. 02, 1995 - Jan 30, 2001). http://nsidc.org/data/docs/daac/ nsidc0002_ssmi_seaice.gd.html

Condy P (1976) Results of the third seal survey in the King Haakon VII Sea, Antarctica. S Afr J Antarct Res 6:2-8

Condy P (1977) Results of the fourth seal survey in the King Haakon VII Sea, Antarctica. S Afr J Antarct Res 7:10-13

Eklund C, Atwood E (1962) A population study of Antarctic seals. J Mammal 43:229-238

Erickson A, Bledsoe L, Hanson M (1989) Bootstrap correction for diurnal activity cycle in census data for Antarctic seals. Mar Mammal Sci 5:29-56

Erickson A, Gilbert J, Otis J (1973) Census of pelagic seals off the Oates and George V Coasts, Antarctica. Antarct J US 8:191-194

Erickson A, Hanson M (1990) Continental estimates and population trends of Antarctic ice seals. In: Kerry K, Hemper G (eds) Antarctic ecosystems: ecological change and conservation. Springer, New York, pp 253-264

Forcada J, Trathan P, Boveng P, Boyd I, Burns J, Costa D, Fedak M, Rogers T, Southwell C (2012) Responses of Antarctic pack-ice seals to environmental change and increasing krill fishing. Biol Conserv 149:40-50

Nordøy E, Blix A (2009) Movement and dive behaviour of two leopard seals (Hydrurga leptonyx) off Queen Maud Land, Antarctica. Polar Biol 32:269-270

Nordøy E, Folkow L, Blix A (1995) Distribution and diving behaviour of crabeater seals (Lobodon carcinophagus) off Queen Maud Land. Polar Biol 15:261-268 
Plötz J, Steinhage D, Bornemann H (2011a) Seal census raw data during campaign EMAGE-I. doi:10.1594/PANGAEA.760097

Plötz J, Steinhage D, Bornemann H (2011b) Seal census raw data during campaign EMAGE-II. doi:10.1594/PANGAEA.760098

Plötz J, Steinhage D, Bornemann H (2011c) Seal census raw data during campaign EMAGE-III. doi:10.1594/PANGAEA.760099

Plötz J, Steinhage D, Bornemann H (2011d) Seal census raw data during campaign EMAGE-IV. doi:10.1594/PANGAEA.760100

Plötz J, Steinhage D, Bornemann H (2011e) Seal census raw data during campaign EMAGE-V. doi:10.1594/PANGAEA.760101

R Development Core Team (2016) R: A Language and Environment for Statistical Computing. R Foundation for Statistical Computing, Vienna, Austria. http://www.R-project.org/

Reeves R, Stewart BS (2003) Marine mammals of the world: An introduction. In: Nowak R (ed) Walker's marine mammals of the world. Johns Hopkins University Press, Baltimore, pp 1-64

Schwarz GE (1978) Estimating the dimension of a model. Ann Stat 6:461-464

Southwell C, Bengtson J, Bester M, Blix A, Bornemann H, Boveng P, Cameron M, Forcada J, Laake J, Nordøy E, Plötz J, Rogers T, Steinhage D, Stewart B, Trathan P (2012) A review of data on abundance, trends in abundance, habitat utilisation and diet for Southern Ocean ice-breeding seals. CCAMLR Sci 19:49-74

Southwell C, Kerry K, Ensor P (2005) Predicting the distribution of crabeater seals Lobodon carcinophaga off east Antarctica during the breeding season. Mar Ecol Prog Ser 299:297-309

Southwell C, Paxton C, Borchers D, Boveng P, De La Mare W (2008a) Taking account of dependent species in management of the Southern Ocean krill fishery: estimating crabeater seal abundance off east Antarctica. J Appl Ecol 45:622-631
Southwell C, Paxton C, Borchers D, Boveng P, Nordøy E, Blix A, De La Mare W (2008b) Estimating population status under conditions of uncertainty: the Ross seal in East Antarctica. Antarct Sci 20:123-133

Southwell C, Paxton C, Borchers D, Boveng P, Rogers T, de la Mare W (2008c) Uncommon or cryptic? Challenges in estimating leopard seal abundance by conventional but state-of-the-art methods. Deep-Sea Res Pt I 55:519-531

Turner J, Barrand N, Bracegirdle T, Convey P, Hodgson D, Jarvis M, Jenkins A, Marshall G, Meredith M, Roscoe H et al (2015) Antarctic climate change and the environment: an update. Polar Rec 50:237-259

Turner J, Bindschadler R, Convey P, DiPrisco G, Fahrbach E, Gutt J, Hodgson D, Mayewski P, Summerhayes C (2009) Antarctic climate change and the environment. Scientific Committee on Antarctic Research, Scott Polar Research Institute, Cambridge

Venables WN, Ripley BD (2002). Modern Applied Statistics with S. Fourth edition. Springer, New York. http://www.stats.ox.ac.uk/ pub/MASS4

Ver Hoef J, Cameron M, Boveng P, London J, Moreland E (2014) A spatial hierarchical model for abundance of three ice-associated seal species in the eastern Bering Sea. Stat Method 17:46-66

Ver Hoef J, London J, Boveng P (2010) Fast computing of some generalized linear mixed pseudo-models with temporal autocorrelation. Comput Stat 25:39-55

Zuur A, Ieno E, Walker N, Saveliev A, Smith G (2009) Mixed effects models and extensions in ecology with R. Springer, New York 